\title{
On the Income Mobility of Rural and Urban Residents in China: 1988 2008
}

\author{
Luo Feng \\ Business School, Foshan University, P.R. China \\ luofeng189@sina.com
}

\begin{abstract}
This paper analyzes the characters of income mobility for both urban and rural residents in China from 1988 to 2008. As a whole, the trend in income mobility is rising for urban residents, but falling for rural residents. For high and low income groups, urban residents' immobility ratio is falling, but rural residents' ratio is rising. For middle income group, urban residents' immobility ratio is rising, but rural residents' ratio is falling. Although the improvement of the poorest and richest $10 \%$ group is pronounced both for urban and rural residents, the immobility ratio for the poorest $10 \%$ group in urban area and the richest $10 \%$ group in rural area is still too high.
\end{abstract}

Index Terms - Income Mobility, Income Transition Matrix, Immobility ratio

\section{Introduction}

Driven by high economic growth, the rapid expansion in income gap among regions and groups has become a major topic of discussion in China. Per capital GDP in China moved above $\$ 3313$ and China became a member of the middle income countries in 2008 (per capital income in 2010 moved to $\$ 4736$ ), but the Gini coefficient in 2010 increased to 0.47 , almost 2 times that of 30 years ago. With growth, China has become one of the most polarized countries in the world, especially as far as urban-rural income gaps are concerned. International experience indicates that middle income countries can fall into a middle income trap, when fully competitive market mechanisms do not emerge, and this can result in social instability. In China where there has been little chance in the relative income position for the poor; rigid hierarchy is a heritage of Chinese political and economic institutions. This underpins a large difference between urban and rural residents. How to improve social mobility and complete competitive market mechanisms remain as key problems for China today.

But because of the relative absence of longitudinal data, relatively little literature studies income mobility in China, what is available can be divided into three categories: (1) Literatures on income mobility in rural China. Most of the results show peasants' income movement speeding up and the hierarchy changing with the transformation of market institutions[1] [3];(2) Literatures on income mobility in urban China, but the studies produce results which in key areas are inconstant[4] [5]; (3) Literatures that focus on the differences between urban and rural residents based on the Chinese Household Nutrition Survey (CHNS), and the results show that income mobility reduces the income gap between rural and urban residents in the long run[6] [8].
Most of these literatures analyze characteristics and trends in income mobility mainly based data before 2004 . Also, since the conclusions of one study do not always accord with others because of the use of different data sets. So, this paper constructs mobility measures and analyzes trends underlying income mobility in China where emerges sharp differences between urban and rural areas using data from CHNS. We also discuss policy implications for further reform in China.

\section{Methodology}

\subsection{Measuring income mobility}

In contrast to income inequality, income mobility deals with changes in income position from one time period or generation to another. These studies usually take place either in an intragenerational context by means of longitudinal or retrospective data for the same individual or in intergenerational contexts where the focus is on the relationship between the incomes of the parents and of their offspring. Although the mobility literature does not provide a unified discourse of analysis, income transition matrix is the dominant method for analyzing income mobility. This captures movement among income ranges. Atkinson(1992) summarized the normal transition matrix as a bi-stochastic matrix. $\mathrm{He}$ considered an income transition matrix from time $t$ to $t+1$, which can be written as:

$$
P(x, y)=\left[p_{i j}(x, y)\right] \in R_{+}^{m \times m}
$$

Where, $x$ and $y$ are income ranges at time $t$ and $t+1$ respectively, and $p_{i j}(x, y)$ is the percentage of the households that in the ith income range at time $t$ move to the $j$ th range at time $t+1 . m$ is the number of orderings in the income distribution, which can be set by the researcher who constructs various quantiles. Every element in the transition matrix is a probability and its value belongs to [0,1]. If the values on the main diagonal are large this means that the probability of remaining in the same income level is large, which also means that income mobility is slow. The basic assumption of Bistochastic Matrix is that the transition probability satisfies a one-order Markov process. Based on the income transition matrix, we can compute indexes reflecting income mobility, such as an immobility ratio, a sub-immobility ratio, a $\chi^{2}$ index etc. If the ordering of this matrix is constructed on a $5 * 5$ ordering basis, the immobility ratio is: 


$$
\frac{1}{5} \sum_{j=1}^{5} P_{j j}
$$

The Sub-immobility ratio is given by the sum of the element of main diagonal and its up-down neighbor elements.

$$
\frac{1}{5} \sum_{i=1}^{5} \sum_{j=i-1}^{i+1} p_{i j}
$$

Use of the income transition matrix is the method to study mobility that received least criticism in the literature.

This paper uses the income transition matrix and immobility ratios to measure relative mobility between urban and rural residents. Most literature analyzes income mobility using 5 quintiles. In order to investigate the features of income mobility in detail, especially the income mobility of the poorest and the richest group, we divide income distributions into 6 groups. Using this grouping, we analyze income mobility using matrix and immobility ratios. Under this grouping, the income transition matrix does not satisfy a oneorder Markov process and the immobility ratio should be calculated using general weighted form,

$$
\sum_{i, j=1}^{n} X_{i} P_{i j}
$$

Where, $\mathrm{X}$ is the weight and represents the population percentage of every group in total population. $P_{j j}$ is the percentage of the households that in $j t h$ income range at time $t$ that stay in $j t h$ range at time $t+1 . n$ is the number of orderings in the income distribution. The advantage of this general weighted form is that it can calculate the immobility ratio no matter how the groups are divided.

\subsection{Data}

This paper analyzes income mobility in China using the CHNS data. CHNS is an ongoing international collaborative project between the Carolina Population Center at the University of North Carolina at Chapel Hill and the National Institute of Nutrition and Food Safety at the Chinese Center for Disease Control and Prevention. The survey covers 8(9) provinces that vary substantially in geography, economic development, public resources, and health indicators. A multistage, random cluster process is used to draw the sample surveyed in each of the provinces. Counties in the 9 provinces are stratified by income (low, middle, and high) and a weighted sampling scheme is used to randomly select 4 counties in each province. In addition, the provincial capital and a lower income city are selected where feasible. In two provinces, other large cities had to be selected. Villages and townships within the counties and urban and suburban neighborhoods within cities are selected randomly. Between 1989 and 2009, this project completed eight surveys, in the years 1989, 1991, 1993, 1997, 2000, 2004, 2006 and 2009 respectively. There are about 3800 households, covering 16000 persons in every survey. There are 8 provinces
Guizhou, Guangxi, Henan, Hubei, Hunan, Jiangshu, Liaoning and Shandong among the survey between 1989 1993. Liaoning was substituted by Helongjiang in 1997 survey. Since 2000, the survey has included 9 provinces, including Guizhou, Guangxi, Heilongjiang, Henan, Hubei, Hunan, Jiangshu, Liaoning and Shandong.

In this study, the household (as opposed to the individual) is the unit of analysis, for in Chinese society the household serves as the production and accounting unit. Family member's labor supply decisions are joint across members of the household based on family background and family member's status. According to our requirement of mobility analysis, the sampling household must appear in every survey year, so we drop the household with missing values in the income variable for one of the survey years. Finally, only 324 urban households and 1397 rural households are included in our analysis. All income data are deflated using the CPI. In order to assess the impact of marketization on income mobility, we first divide the transition era into 3 periods according to key points in China's reforms. During the economic transition, 1992 is important for the reform process, since Dengxiaoping's South Tour Speeches and Convocation of the 14th National Congress of party urged building a socialist market economy and markets began to further develop. The marketization process which followed as different, especially until 2000. For example, the Law of Securities was published in 1999; Southern, Eastern and Northern labor information nets were build respectively in 2000; A land market begins to operate in 2000. This paper divides the transition era into 3 periods : 1989 1993(T1), 1993 2000(T2), 2000 2009(T3), and we label T1-T2 prereform period and T2-T3 the postreform period.

\section{Empirical Results}

Tables 1 and 2 present income transition matrices for urban residents for the T1-T2 and T2-T3 periods, and Table 3 and 4 are the income transition matrices for rural residents. Based on Tables 1-4, we can compute various income mobility indices (see Table 5).

\subsection{The characters of income mobility for urban residents}

During T1-T2, the average immobility ratio of urban residents was $31.29 \%$, i.e. nearly one third urban families did not change their position. For every group, Q1's immobility ratio was the highest for all groups, reaching $42.42 \%$, i.e. $42.42 \%$ did not change their position at P1-P2 period which shows the larger immobility that occurred in the lowest income rank group. Group Q2 and Q6's immobility ratio was the lowest among all groups, respectively $20.83 \%$ and $21.21 \%$, which implies that these two groups have more high mobility. Q3 and Q4 have the same immobility ratio and both reached $32.1 \%$. But they had a reversed mobility direction: group Q3 had more upward mobility than Q4, which means that the lower income group had a chance to improve their income position. Q5's immobility ratio is $39.58 \%$, lower only than Q1, which means that Q5's income position is more stable. 
During T2-T3, the average immobility ratio of urban residents falls to $29.4 \%$. Q1 、 Q5 - Q6's immobility ratios also all fall, respectively by $9.1 \% \cdot 10.4 \%$ and $3.0 \%$, but Q2、Q3 and Q4's immobility ratios rise slightly. Specifically, Q5's upward mobility ratio rises by $16.77 \%$. This suggests that the income mobility of urban residents improved, because the whole immobility ratio fall. The lowest group (Q1)shows an upward trend and the richest group (Q5 and Q6)a downward trend. If this trend were to continue, the income gap between urban families would disappear.

\subsection{The characters of Income mobility for rural residents}

During T1-T2, the average immobility ratio of rural residents reaches $28.42 \%$ what was lower than that of urban residents. This suggests that rural residents had more opportunity to change their income position. For each group, Q1's income immobility ratio was only $21.58 \%$, the lowest of all groups. Q6's income immobility ratio was the highest across all groups reaching to $39.57 \%$. Q2's immobility ratio was $22.86 \%$, and its downward mobility ratio was $16.19 \%$ and upward mobility ratio was $60.95 \%$; Q5's immobility ratio was $25.71 \%$, and its downward ratio was $55.23 \%$ and upward ratio was $19.05 \%$. This suggests that Q2 and Q5 had higher income mobility, while Q3 and Q4 close to average income mobility and the lower group's status was more stable.

During T2-T3, the average immobility ratio of rural residents rose to $30.21 \%$, higher by about $1 \%$ compared to periods $\mathrm{T} 1-\mathrm{T} 2$, and approximately the same as the immobility ratios of urban residents. Q1 and Q6's immobility ratios all fall, reaching $18.71 \%$ and $35.97 \%$ respectively. Q1 is still the lowest and Q6 is the highest across all groups which imply that the poor had more chance to improve their income position and the richest rank's immobility was decreasing. Q2 and Q5's immobility ratio rose to $28.57 \%$ and $33.33 \%$ respectively. Q3 and Q4's immobility ratio moved in opposite directions, Q3's ratio fell to $32.29 \%$ and Q4's ratio rose to $29.51 \%$.

As a whole, the trend in income mobility is increasing for urban residents, but falling for rural residents. For high and low income group, urban residents' immobility ratio is falling, but rural residents' ratio is rising. For middle income group, urban residents' immobility ratio is rising, but rural residents' ratio is falling. Although the immobility ratio of the poorest and richest $10 \%$ group are falling both for urban and rural residents, the solidification for the poorest $10 \%$ group in urban area and the richest $10 \%$ group in rural area is still rigorous.

TABLE 1. Income Transition Matrix of Urban Residents from T1 to T2 (Unit: \%)

\begin{tabular}{|c|c|c|c|c|c|c|c|}
\hline \multicolumn{8}{|c|}{ T2's position } \\
\hline \multirow{7}{*}{ T1's position } & & $\mathrm{Q} 1$ & $\mathrm{Q} 2$ & Q3 & $\mathrm{Q} 4$ & Q5 & Q6 \\
\hline & Q1 & 42.42 & 24.24 & 15.15 & 9.09 & 3.03 & 6.06 \\
\hline & $\mathrm{Q} 2$ & 14.58 & 20.83 & 39.58 & 14.58 & 8.33 & 2.08 \\
\hline & Q3 & 7.41 & 13.58 & 32.1 & 28.4 & 7.41 & 11.11 \\
\hline & $\mathrm{Q} 4$ & 4.94 & 17.28 & 19.75 & 32.1 & 13.58 & 12.35 \\
\hline & Q5 & 2.08 & 4.17 & 18.75 & 27.08 & 39.58 & 8.33 \\
\hline & Q6 & 3.03 & 9.09 & 18.18 & 27.27 & 21.21 & 21.21 \\
\hline
\end{tabular}

Source: Calculated and compiled by authors and original dates come from CHNS.

TABLE 2. Income Transition Matrix of Urban Residents from T2 to T3 (Unit: \%)

\begin{tabular}{|c|c|c|c|c|c|c|c|}
\hline \multicolumn{8}{|c|}{ T3's position } \\
\hline \multirow{7}{*}{ T2's position } & & Q1 & Q2 & Q3 & $\mathrm{Q} 4$ & Q5 & Q6 \\
\hline & Q1 & 33.33 & 42.42 & 18.18 & 3.03 & 3.03 & 0 \\
\hline & Q2 & 14.58 & 22.92 & 31.25 & 22.92 & 4.17 & 4.17 \\
\hline & Q3 & 12.35 & 17.28 & 33.33 & 24.69 & 7.41 & 4.94 \\
\hline & $\mathrm{Q} 4$ & 6.17 & 4.94 & 19.75 & 35.8 & 22.22 & 11.11 \\
\hline & Q5 & 0 & 6.25 & 16.67 & 22.92 & 29.17 & 25 \\
\hline & Q6 & 0 & 6.06 & 27.27 & 27.27 & 21.21 & 18.18 \\
\hline
\end{tabular}

Source: Calculated and compiled by authors and original dates come from CHNS.

TABLE 3. Income Transition Matrix of Rural Residents from T1 to T2 (Unit: \%)

\begin{tabular}{|c|c|c|c|c|c|c|c|}
\hline \multicolumn{8}{|c|}{ T2'position } \\
\hline \multirow{7}{*}{ T1'position } & & Q1 & $\mathrm{Q} 2$ & Q3 & Q4 & Q5 & Q6 \\
\hline & Q1 & 21.58 & 26.62 & 32.37 & 13.67 & 4.32 & 1.44 \\
\hline & Q2 & 16.19 & 22.86 & 26.19 & 26.19 & 6.19 & 2.38 \\
\hline & Q3 & 12 & 13.43 & 35.14 & 27.43 & 9.43 & 2.57 \\
\hline & Q4 & 6.3 & 14.04 & 24.93 & 27.79 & 18.91 & 8.02 \\
\hline & Q5 & 4.76 & 10 & 13.33 & 27.14 & 25.71 & 19.05 \\
\hline & Q6 & 0.72 & 5.76 & 8.63 & 17.99 & 27.34 & 39.57 \\
\hline
\end{tabular}

Source: Calculated and compiled by authors and original dates come from CHNS. 
TABLE 4. Income Transition Matrix of Rural Residents from T2 to T3 (Unit: \%)

\begin{tabular}{|c|c|c|c|c|c|c|c|}
\hline \multicolumn{8}{|c|}{ T3'position } \\
\hline \multirow{7}{*}{ T2'position } & & Q1 & Q2 & Q3 & Q4 & Q5 & Q6 \\
\hline & Q1 & 18.71 & 25.9 & 33.09 & 19.42 & 2.16 & 0.72 \\
\hline & Q2 & 16.19 & 28.57 & 28.57 & 20 & 5.71 & 0.95 \\
\hline & Q3 & 10.86 & 13.43 & 32.29 & 29.71 & 9.14 & 4.57 \\
\hline & Q4 & 9.17 & 11.75 & 23.5 & 29.51 & 16.33 & 9.74 \\
\hline & Q5 & 2.38 & 10.95 & 16.67 & 19.52 & 33.33 & 17.14 \\
\hline & Q6 & 2.88 & 2.16 & 10.07 & 23.02 & 25.9 & 35.97 \\
\hline
\end{tabular}

Source: Calculated and compiled by authors and original dates come from CHNS.

TABLE 5. Rural and Urban Residents' Income Mobility (\%)

\begin{tabular}{|c|c|c|c|c|c|}
\hline & \multirow{2}{*}{ Category } & \multicolumn{2}{|c|}{ Urban } & \multicolumn{2}{|c|}{ Rural } \\
\hline & & $\mathrm{T} 1-\mathrm{T} 2$ & T2-T3 & $\mathrm{T} 1-\mathrm{T} 2$ & $\mathrm{~T} 2-\mathrm{T} 3$ \\
\hline \multirow{7}{*}{ Level of mobility } & Total immobility & 31.29 & 29.4 & 28.42 & 30.21 \\
\hline & Q6's immobility & 21.21 & 18.18 & 39.57 & 35.97 \\
\hline & Q5's immobility & 39.58 & 29.17 & 25.71 & 33.33 \\
\hline & Q4's immobility & 32.1 & 35.8 & 27.79 & 29.51 \\
\hline & Q3's immobility & 32.1 & 33.33 & 35.14 & 32.29 \\
\hline & Q2's immobility & 20.83 & 22.92 & 22.86 & 28.57 \\
\hline & Q1's immobility & 42.42 & 33.33 & 21.58 & 18.71 \\
\hline \multirow{7}{*}{$\begin{array}{c}\text { Dynamic of income } \\
\text { position }\end{array}$} & Total up/down mobility ratio & 97.56 & 110.76 & 110.97 & 112.45 \\
\hline & Q6's down ratio & 37.80 & 40.36 & 29.84 & 32.27 \\
\hline & Q5's up/down ratio & 15.99 & 54.54 & 34.49 & 34.61 \\
\hline & Q4's up/down ratio & 61.78 & 108.00 & 59.49 & 58.69 \\
\hline & Q3's up/down ratio & 223.54 & 125.01 & 155.05 & 178.76 \\
\hline & Q2's down/up ratio & -22.58 & -23.32 & -26.56 & -29.31 \\
\hline & Q1's up ratio & 28.31 & 29.69 & 34.89 & 36.43 \\
\hline
\end{tabular}

Source: Calculated and compiled by authors and original dates come from CHNS.

\section{Conclusions}

This paper analyzes the characteristics of income mobility between urban and rural residents in China during the whole of the transition era using income transition matrices and immobility ratio indices. This paper also assesses factors influencing income mobility between urban and rural residents, focusing especially on the two mechanism of allocating resources: Market as provided by educational attainment and redistributive proxied by cadre identification and vocational position.

The results show that there have adverse mobility trends between urban and rural areas as a whole. Income mobility is rising fast for urban residents, but is falling for rural residents. For high and low income group, urban residents' immobility ratio is falling, but rural residents' ratio is rising. For middle income group, urban residents' immobility ratio is rising, but rural residents' ratio is falling. Although the immobility ratio of the poorest and richest 10\% group are falling both for urban and rural residents, the solidification for the poorest $10 \%$ group in urban area and the richest $10 \%$ group in rural area is still rigorous.

\section{Acknowledgement}

The author is grateful to the Ontario Research Fund for financial support and to a seminar group at UWO for comments and discussions.

\section{References}

[1] Sun Wenkai, Lu Jiangyong and Bai Chongen. "An Analysis of Income Mobility in Rural China". Economic Research Journal, vol.8, pp.43-57, 2007.

[2] Xuehua S, Alexander J N and Xian X. "Household Income Mobility in Rural China:1989-2006”.Economic Modelling,vol.5,pp.1090-1096,2010.

[3] Zhang Qi, Mi Jianwei and Huang Jikun. "Income Mobility and Income Distribution: Evidence from Rural China". Economic Research Journal, vol.11, pp.123-138, 2007.

[4] Khor N and Pencavel J. "Income Mobility of Individuals in China and The United States", Economics and Transition, vol.3, pp.417-458, 2006.

[5] Yin Heng, Li Shi and Deng Quheng. "Income Mobility in Urban China", Economic Research Journal, vol.10, pp.30-43, 2006.

[6] Wang Haigang. "The Household Income Mobility and Its Equalizing Long-term Income in China". Economic Research Journal, vol.1, pp.6570, 2005.

[7] Wang Chaoming and Hu Qizhi, "Income Mobility Empirical Researchbased on a Variety of Indicators Measure", Management World, vol.10, pp.30-40, 2008.

[8] Zhouxin and Wan Fang. "Income Mobility, Income Gap and Social Welfare of Urban and Rural Residents in China". Management World, vol.5, pp.65-74, 2010. 